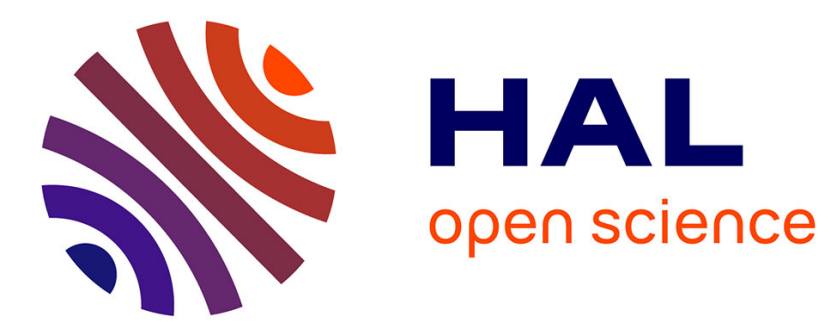

\title{
Structuring the three electric field components of light
}

\author{
F. Maucher, Stefan Skupin, S. Gardiner, I. G Hughes
}

\section{To cite this version:}

F. Maucher, Stefan Skupin, S. Gardiner, I. G Hughes. Structuring the three electric field components of light. 2019 Conference on Lasers and Electro-Optics Europe \& European Quantum Electronics Conference (CLEO/Europe-EQEC), Jun 2019, Munich, Germany. pp.1-1, 10.1109/CLEOEEQEC.2019.8871683 . hal-02327610

\section{HAL Id: hal-02327610 https://hal.science/hal-02327610}

Submitted on 22 Oct 2019

HAL is a multi-disciplinary open access archive for the deposit and dissemination of scientific research documents, whether they are published or not. The documents may come from teaching and research institutions in France or abroad, or from public or private research centers.
L'archive ouverte pluridisciplinaire HAL, est destinée au dépôt et à la diffusion de documents scientifiques de niveau recherche, publiés ou non, émanant des établissements d'enseignement et de recherche français ou étrangers, des laboratoires publics ou privés. 


\title{
Structuring the three electric field components of light
}

\author{
F. Maucher ${ }^{1}$, S. Skupin ${ }^{2}$, S. A. Gardiner ${ }^{3}$, I. G. Hughes ${ }^{3}$ \\ 1. Department of Physics and Astronomy, Aarhus University, Ny Munkegade 120, 8000 Aarhus C, Denmark \\ 2. Univ. Lyon, Université Claude Bernard Lyon 1, CNRS, Institut Lumière Matière, F-69622, Villeurbanne, France \\ 3. Joint Quantum Centre (JQC) Durham-Newcastle, Department of Physics, Durham University, Durham DH1 3LE, United Kingdom
}

Unless the beam's transverse electric field components are divergence-free in the two-dimensional transverse plane [1], tightly focused light typically leads to a non-negligible longitudinal electric field component [2], where the terms longitudinal and transverse electric field components refer to the components of the electric field that are parallel or perpendicular, respectively, to the direction of the mean Poynting flux. Having a longitudinal electric field component does not add a new degree of freedom, in the sense that all components of the electric and magnetic fields are still fixed by prescribing two electric field components in a plane. However, it is the electric field component parallel to the direction of the Poynting flux that makes it somewhat special.

In this contribution we investigate simultaneous structuring of all three electric field components, and in particular we demonstrate that complex light shaping of the longitudinal component is indeed possible. For the proposed algorithm, a two-dimensional Helmholtz decomposition of the transverse electric field components in the transverse plane is key. The Helmholtz decomposition allows the generalization of the notion of "radial" and "azimuthal" polarization in the following way: radial polarization corresponds to an electric field that is "curl-free" in the transverse plane - which as we shall see can be interpreted as a flow solely due to sinks and sources without vorticity - and is the part of the field that gives rise to the longitudinal polarization. An azimuthally polarized electric field is "divergence-free" in the transverse plane - analogously to a flow solely due to vorticity without any sinks or sources - and does not give rise to any longitudinal electric field component. Employing those polarization states turns out to be very useful for computing numerically exact solutions to Maxwell's equations with structured electric field components, and moreover facilitates an intuitive understanding of tightly focused vector beams through straightforward analogies to electrostatics, magnetostatics and fluid dynamics.
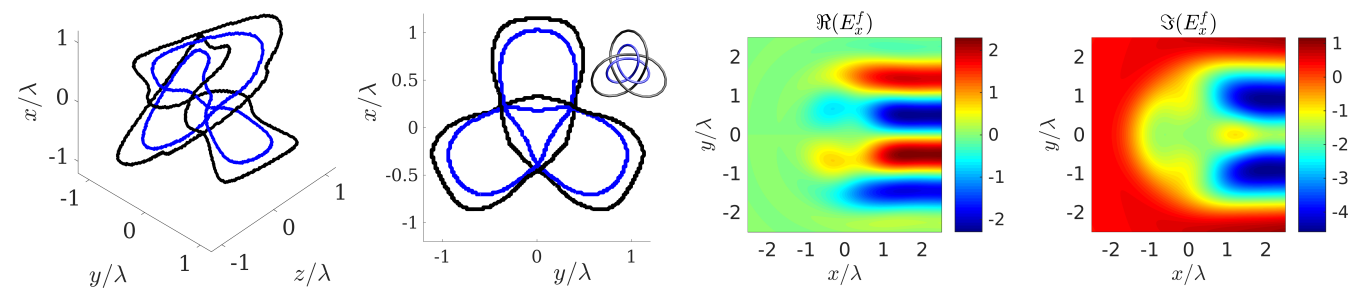

Fig. 1 Vortex lines forming two linked trefoils in different components of the electric field: The blue line represents the vortex line in the transverse component $E_{y}$, the black line the vortex line in the longitudinal component $E_{z}$. The corresponding (semi-infinite) profiles in $E_{x}$ at $z=0$ (focus) are shown in the right panels.

In Fig. 1 we demonstrate the tremendous capabilities of our algorithm by creating knotted vortex lines in the longitudinal and one transverse electric field component $\left(E_{y}\right)$ simultaneously. The topology of the two knots we envisage is sketched in the two left panels: the vortex lines of the $E_{y}$ and $E_{z}$ components form two linked trefoil knots. Even though at first glance this example may seem somewhat contrived, it highlights that vortex lines in different electric field components can be chosen to be arbitrarily close without reconnecting. This is a priori not possible for vortex lines in a single electric field component, which could be of importance when considering inscribing these vortex lines onto matter. The corresponding transverse electric field component $E_{x}$, which is fully determined by our algorithm and shown in the right panels of Fig. 1, is formally nonzero on a semi-infinite interval, and has to be attenuated in practise by, e.g., a sufficiently wide super-Gaussian profile. We checked that such procedure does not affect the propagation of the components of interest close to the optical axis.

We believe that our findings will broaden the range of accessible vector beams extensively and trigger further theoretical and experimental investigations involving structured light.

\section{References}

[1] F. Maucher, S. Skupin, S. A. Gardiner, and I. G. Hughes, "Creating complex optical longitudinal polarization structures," Phys. Rev. Lett. 120, 163903 (2018).

[2] B. Richards and E. Wolf, "Electromagnetic diffraction in optical systems. ii. structure of the image field in an aplanatic system," Proceedings of the Royal Society of London A: Mathematical, Physical and Engineering Sciences 253, 358-379 (1959). 